\title{
Differences in Design Considerations between InGaN and Conventional High-Brightness Light-Emitting Diodes
}

\author{
Song Jae Lee \\ Electronics Engineering Department, Chungnam National University Yusung-Gu Koong-Dong 220 Taejon, 305-764, KOREA
}

(Received: October 8, 1997)

\begin{abstract}
Based on the escape cone concepts, high-brightness light-emitting diodes (LEDs) have been analyzed. In AlGaAs or InGaAIP LEDs, photon absorption in the ohmic region under the electrode is known to be significant. Thus, in general, a thick window layer (WL) and a transparent substrate (TS) would minimize photon shielding by the electrodes and considerably improve photon output coupling efficiency. However, the schemes do not seem to be necessary in InGaN system. Photon absorption in ohmic contact to a wide bandgap semiconductor such as GaN may be negligible and, as a result, the significant photon shielding by the electrodes will not degrade the photon output coupling efficiency so much. The photon output coupling efficiency estimated in InGaN LEDs is about $2.5-2.8$ times that of the conventional high-brightness LED structures based on both WL and TS schemes. As a result, the external quantum efficiency in InGaN LEDs is as high as $9 \%$ despite the presumably very low internal quantum efficiency.
\end{abstract}

\section{INTRODUCTION}

Over the years the research emphasis in lightemitting diodes (LEDs) has been focused on growth of various light-emitting materials rather than on development of particular structures. Recently, however, as the demand for high-brightness LEDs increases rapidly since blue LEDs were commercialized to clear the way for outdoor full-color displays[1-4], the need to develop structures suited for increased brightness has become stronger than ever. LEDs in general have a relatively simple geometry as shown in Fig. 1, and it may have been thought that the device can be designed intuitively based on simple conceptual understanding. Consequently literature on theoretical analysis or design of LEDs has been very rare.[5-8] Fig. 2 shows schematic diagrams of some of the important highbrightness LED structures developed thus far. The structure in Fig. 2(a) is well established for AlGaAs LEDs emitting in red spectrum. In this structure, the absorbing substrate(AS) is replaced by a thick lower confining layer of a high bandgap energy often called the transparent substrate (TS). And the scheme helps to improve the photon output coupling efficiency. The ohmic region is also known to be usually very lossy due to the amorphous nature of the crystal therein. Thus, similarly a thick upper confining layer of high bandgap energy, often called the window layer (WL), minimizes the photon shielding by the top electrode and improves the light emitting efficiency.

The WL and TS schemes, however, can be imple-

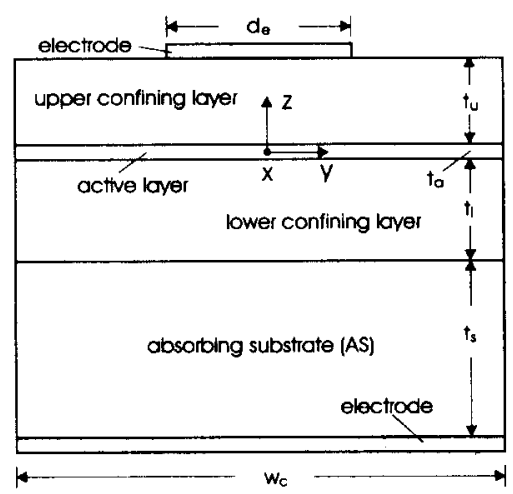

(a)

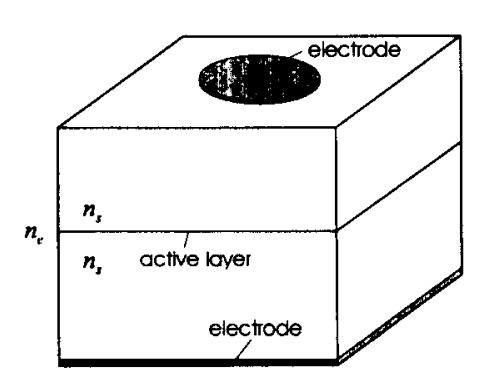

(b)

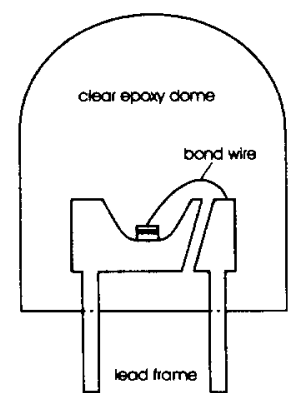

(c)
FIG. 1. (a) Standard LED structure, (b) Simplified LED chip model, (c) LED lamp.

mented relatively easily only in red AlGaAs LEDs that are usually grown by liquid phase epitaxy (LPE) with a relatively large crystal growth rate. Both WL and TS should typically be as thick as $50 \mu \mathrm{m}$. In the case of 


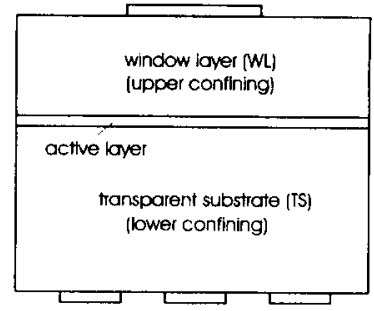

(a)

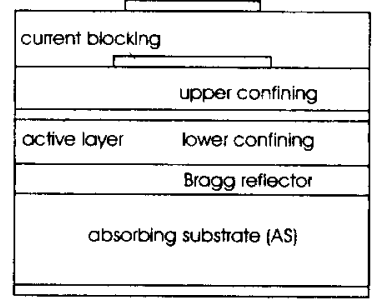

(b)

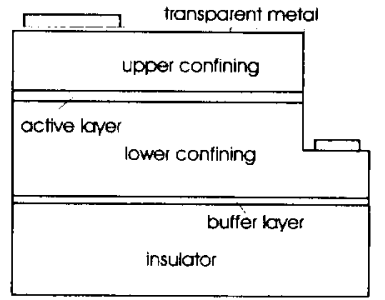

(c)
FIG. 2. High-brightness LED structures. (a) Window layer (WL) and transparent substrate (TS) structure (b) Current blocking layer (CBL) and Bragg reflector (BR) structure (c) InGaN structure.

the InGaAIP LEDs that are usually grown by organometallic vapor phase epitaxy (OMVPE) with a much smaller growth rate, layers as thick as required in WL and TS can hardly be grown. Recently, however, Kish et al. achieved very impressive external quantum efficiencise with InGaAlP LEDs based on both WL and TS schemes, by combining hybrid growth of a thick $\mathrm{GaP}$ WL with a wafer bonding (WB) technique. $[9,10]$ As another approach to minimize the photon absorption in both the substrate and ohmic electrode, a novel structure as schematically shown in Fig. 2(b) has been developed by Sugawara et al.[11] In their structure, the purpose of the current blocking layer (CBL) is to make the current spread to the outer region of the active layer where the effect of photon shielding by the top electrode is minimal. The purpose of the Bragg reflector (BR) is to reflect the photons directed to the lossy substrate back into the chip to improve the external quantum efficiency.

The InGaN LED structure schematically shown in Fig. 2(c) may be an exception in terms of structure design.[1-4] In the structure grown by OMVPE on an insulating sapphire substrate, the lower electrode is formed on the terraced lower confining layer and the thickness of the overall upper confining layers is less than about $1 \mu \mathrm{m}$. Furthermore, a very thin ohmic contact layer is deposited over the whole top surface of the chip.[4] Thus, judging from conventional design concepts, significant numbers of photons directed either upward or laterally would be shielded by the top electrode, possibly leading to a very poor photon output coupling efficiency. Combined with the presumably very low internal quantum efficiency due to a very high density of defects in the InGaN crystal, the exter-

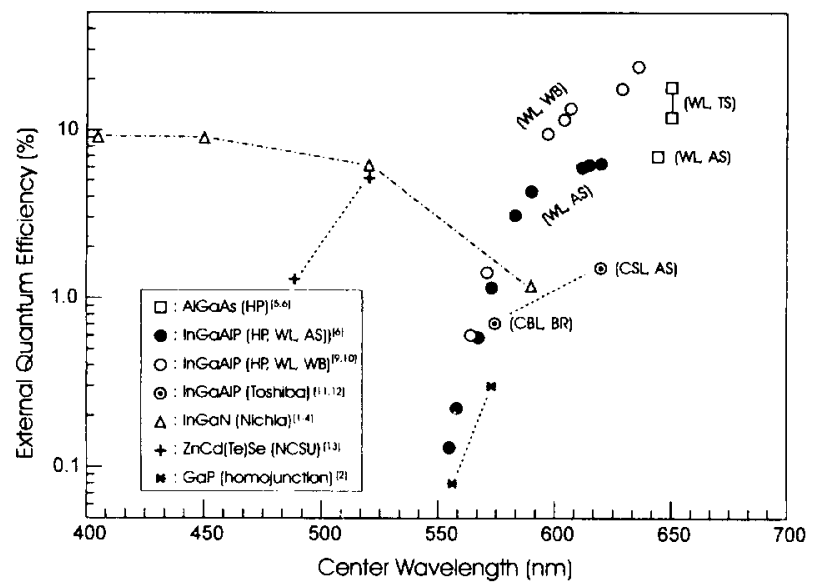

FIG. 3. External quantum efficiencies achieved in high-brightness LEDs developed thus far.

nal quantum efficiency expected should be extremely poor. In reality, however, the external quantum efficiency obtained in InGaN LEDs is as high as about $9 \%$ as shown in Fig. 3 and is comparable to those in high-brightness LEDs based on both the WL and TS schemes. The negligible photon absorption in the sapphire insulator substrate along with the minimal shielding of the laterally directed photons by the lower electrode on the terraced confining layer, may not be enough to explain the remarkably high external quantum efficiency. One important motivation of this paper is to explain the differences in design considerations between InGaN and conventional high-brightness LED structures. Clear understanding of the differences may be essential to optimize LED structures in general for improved brightness or output.

\section{LIGHT-EMITTING EFFICIENCY AND ESCAPE CONE}

Standard LED chips in general consist of various layers as described in Fig. 1(a). However, the refractive index differences between layers are usually small, and thus, all the layers may be assumed to have the same reflective index $n_{s}$ as described in Fig. 1(b), especially when the absorbing substrate (AS) is replaced with the transparent substrate (TS). We will focus, for the time being, on the simplified model even though the model may not be applicable to InGaN LED structure shown in Fig. 2(c).

Photons generated in the active layer are in general randomly directed. Thus, in the output coupling process, some of the photons are either shielded by electrodes or reflected off the chip-wall and absorbed inside the chip. Because the degree of shielding varies with the position in the active layer, the photon output coupling efficiency $\eta_{c}$ would be also a function of position. External quantum efficiency, $\eta_{e x t}$, the most 
important parameter to determine the brightness or output power of LED, is closely related to the photon output coupling efficiency $\eta_{c}(x, y)$ and may be expressed by $[14,15]$

$$
\eta_{\text {ext }}=\frac{\iint_{\text {active }} J(x, y) \eta_{i}(x, y) \eta_{c}(x, y) d x d y}{\iint_{\text {active }} J(x, y) d x d y}
$$

where $\eta_{i}$, and $J$ are the internal quantum efficiency and current density in the active layer, respectively. The z-dependence of $\eta_{i}, \eta_{c}$ and $J$ would be negligible in the case of a relatively thin active layer as assumed here. The internal quantum efficiency $\eta_{i}$ is in general dependent primarily on the material quality of the active layer and may be assumed to be constant through out the active region. Eq. (1) is then reduced to

$$
\eta_{\text {ext }}=\eta_{i} \frac{\iint_{\text {active }} J(x, y) \eta_{c}(x, y) d x d y}{\iint_{\text {active }} J(x, y) d x d y}
$$

The current density $J(x, y)$ will be in general determined by the electrode design and the resistivity of the crystal layers. However, if the confining layers are thick enough as in most high-brightness LEDs, the current will spread significantly rendering the current density J uniform throughout the active layer. Then the external quantum efficiency, $\eta_{\text {ext }}$ will be further reduced to

$$
\eta_{e x t}=\eta_{i} \eta_{c a v g}=\eta_{i} \frac{\iint_{\text {active }} \eta_{c}(x, y) d x d y}{\iint_{\text {active }} d x d y}
$$

where $\eta_{\text {cavg }}$, often called the photon extraction efficiency, is the space average of the photon output coupling efficiency over the whole active region. Since the internal quantum efficiency $\eta_{i}$ in Eq.(3) is in general a material dependent parameter, the most appropriate approach to increase the output power or brightness, in a structure-design point of view, would be to improve the average photon output coupling efficiency $\eta_{\text {cavg }}$.

In general, it is not an easy task to estimate $\eta_{c}(x, y)$. One approach is to employ the Monte Carlo method, [16] in which layer-to-layer trajectories of randomly generated photons are traced stochastically to obtain detailed information of the behavior of photons. In this work, however, the escape cone concepts will be used. We first review the photon reflection and transmission at a dielectric interface as schematically described in Fig. 4(a). In general, the photon reflection or transmission is a function of the incidence angle $\theta_{i}$ and the direction of polarization with respect to the incidence plane. Fig. 4(b) shows the variation of the photon transmittance as a function of the photon incidence angle $\theta_{i}$ for typical cases. An important feature is that the photon transmittance is zero regardless of the polarization when the incidence angle $\theta_{i}$ is larger than the critical angle $\theta_{c}[17]$ (a)
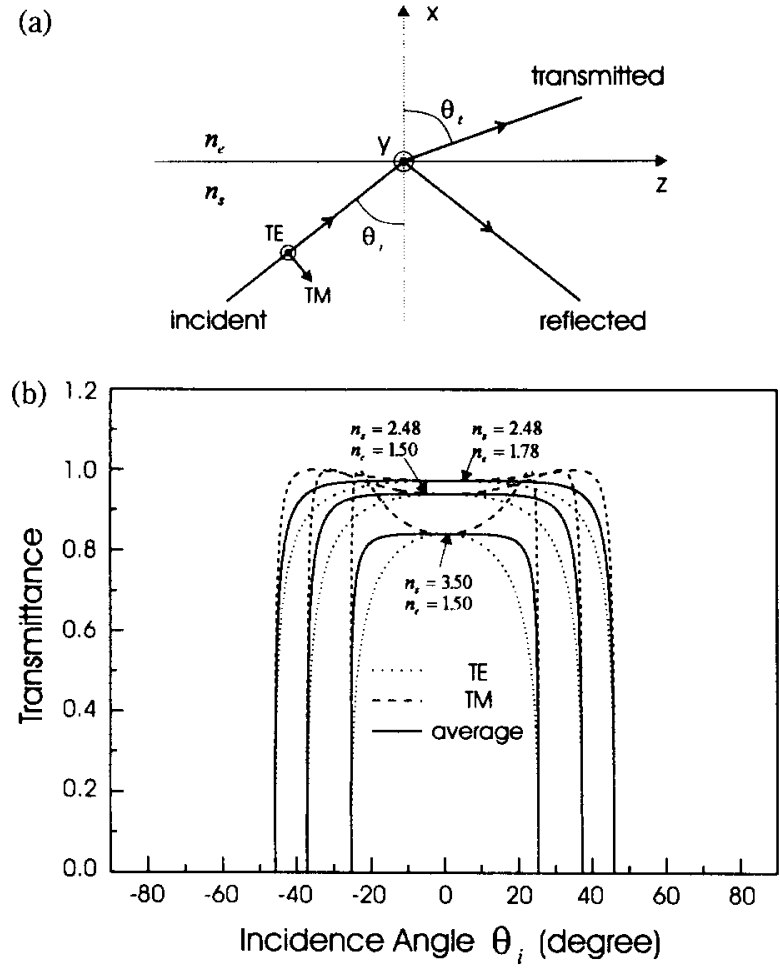

FIG. 4. (a) Photon reflection and transmission model at the semiconductor-encapsulat interface (b) Photon transmittance as a function of the incidence angle and the polarization.

$$
\theta_{c}\left(n_{e}, n_{s}\right)=\sin ^{-1}\left(n_{e} / n_{s}\right)
$$

where $n_{e}$ is the refractive index of the epoxy in which the chip is often encapsulated. In the case of a bare chip, the $n_{e}$ should be replaced by the refractive index of air which is close to unity. It is noted that the transmittance for the transverse magnetic (TM) photons is always larger than that for transverse electric (TE) photons due to the Brewster angle phenomenon.[18] For instance, at the Brewster incidence angle $\theta_{B}$

$$
\theta_{B}=\tan ^{-1}\left(n_{e} / n_{s}\right)
$$

the reflection coefficient for TM photons becomes zero, implying complete transmission of photons. In the case of LED, however, the polarization of photons would be random and thus the average photon transmittance may be assumed to be the same as the average of the transmittance of TE and TM photons.

As a result of the Brewster angle phenomenon, the average photon transmittance within the transmission window between $-\theta_{c}$ and $\theta_{c}$ is relatively flat and leads to the so-called escape cones as illustrated in Fig. 5(a). The escape cones, one for each facet of the chip and with a solid angle $\Omega_{c}$ given by

$$
\Omega_{c}\left(n_{s}, n_{e}\right)=\int_{\phi=0}^{2 \pi} \int_{\theta=0}^{\theta_{c}} \sin \theta d \theta d \phi
$$




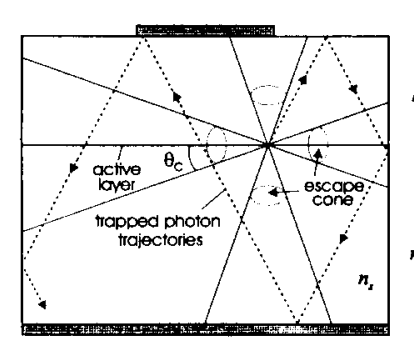

(a)

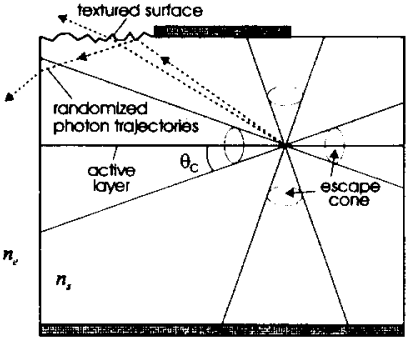

(b)
FIG. 5. Escape cones and photon trajectories inside the chip. (a) Ideal flat surface, (b) Textured surface.

$$
=2 \pi\left(1-\sqrt{1-\left(n_{e} / n_{s}\right)^{2}}\right)
$$

will not overlap the others, because the critical angle $\theta_{c}$ is usually less than $45^{\circ}$ for most practical LEDs. The photons emitted inside the escape cones that are not shielded by the electrodes will avoid the total internal reflection and have a chance to couple out of the chip. In the output coupling process, some of the photons may be either Fresnel-reflected from the coupling facet or total-internally reflected off the side wall. However, the incidence angle with respect to the opposite facet would be the same, and therefore, if internal photon absorption is ignored, virtually all the photons emitted inside the escape cones will eventually couple out of the chip after a couple of flights.

On the other hand, the photons emitted outside the escape cone will always be total-internally reflected back into the chip. Especially when the shape of the chip is of rectangular cubic as assumed here, the photons emitted outside the escape cone once would experience a sequence of total internal reflections as illustrated in Fig. 5(a) to be eventually absorbed inside the chip. One approach to extract some of the photons emitted outside the escape cones is to employ the textured surface as shown in Fig. 5(b), at which some degree of photon trajectory randomization occurs. [7,8]

\section{CONVENTIONAL HIGH-BRIGHTNESS LEDS}

In conventional LED structures based on either AlGaAs or InGaAlP system, both usually grown on GaAs substrate, significant photon absorption occurs in the substrate whose bandgap energy is smaller than the photon energy. The ohmic region under the electrode is also very lossy due to the amorphous nature of the crystal therein.[18] In order to improve the photon output coupling efficiency $\eta_{c}$, photon shielding by the electrodes should be minimized. If the photon trajectory randomization, due to either diffused reflection off the chip wall or to photon absorption/reemission in the active layer, is negligible, it may not be necessary to consider the photons emitted outside escape cones because they would be mostly trapped and absorbed inside the chip regardless of the shielding by electrodes.

Significant differences in photon shielding characteristics exist between vertical and lateral escape cones. Since the details were described elsewhere[14], we will focus only on the key results. The column of a chip whose side walls are free from lossy electrodes can be viewed as a highly multi-moded waveguide for the photons traveling through either upward or downward cone. In this case, average shielding effect of the upward cone would be mostly determined by the ratio of top surface covered by the electrode and will not depend sensitively on the upper confining layer thickness $t_{u}$. Thus the average upward cone output coupling efficiency $\eta_{c u}$ may be estimated by

$$
\eta_{c u}=1-\frac{\pi d_{e}^{2}}{4 w_{c}^{2}}
$$

where $w_{c}$ and $d_{e}$ are the chip size and the diameter of the top circular electrode, respectively. In highbrightness LED structures, the bottom electrode is often patterned as shown in Fig. 2(a). In this case, most of the photons incident on the opened region of the contact would be reflected back into the chip off the usually silver-plated metal lead frame to which the chip is attached. The photons coupled back into the upward cones would be also governed by Eq.(7). Thus, $\eta_{c d}$, the average output coupling efficiency for the downward cone would be similarly estimated by

$$
\eta_{c d}=\frac{A_{o}}{w_{c}^{2}} \eta_{c u}=\frac{A_{o}}{w_{c}^{2}}\left(1-\frac{\pi d_{e}^{2}}{4 w_{c}^{2}}\right)
$$

where $A_{o}$ is the area of the opened region of the bottom electrode. Note that both $\eta_{c u}$ and $\eta_{c d}$ in Eqs.(7) and (8), respectively, are not a function of the upper confining layer thickness $t_{u}$.

In the case of lateral cones, however, the shielding characteristics are quite different. In general, the lateral cones are shielded less as the confining layer thickness increases. As shown in Fig. 6(a), if the upper confining layer thickness $t_{u}$ is chosen by

$$
t_{u}=\frac{w_{c}+d_{e}}{2} \tan \theta_{c}=\frac{w_{c}+d_{e}}{2} \frac{n_{e}}{\sqrt{n_{s}^{2}-n_{e}^{2}}}
$$

all the lateral cones would be cleared from the top electrode regardless of the position in the active layer. Further increase of the upper confining layer thickness would not improve the photon output coupling efficiency substantially. Typical numbers for $w_{c}$ and $d_{e}$ are about $250 \mu \mathrm{m}$ and $100 \mu \mathrm{m}$, respectively. The $t_{u}$ is then evaluated to about $85 \mu \mathrm{m}$ and may be too thick to be implemented in practical LEDs.

It is noted, however, that the relation in Eq.(9) has been obtained based on the meridional cross-sectional view of the chip. In the case of a circular top electrode as assumed in this work, the incidence plane would be 
(a)

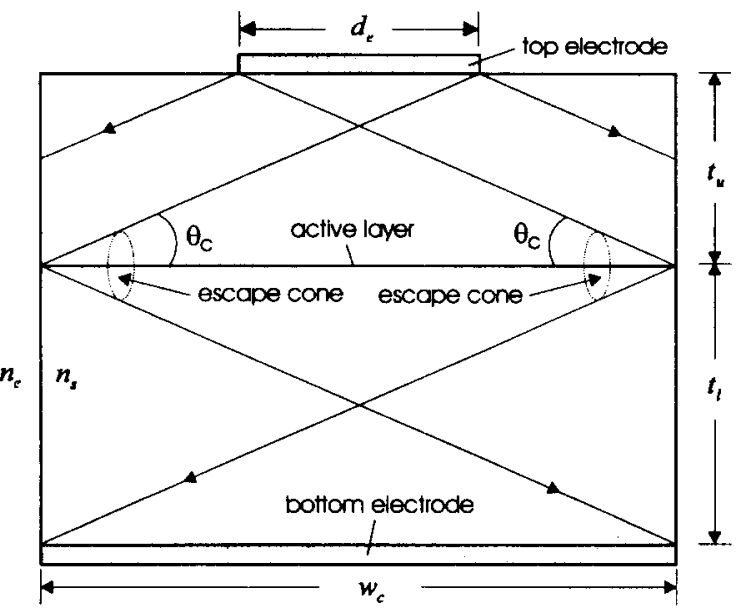

(b)

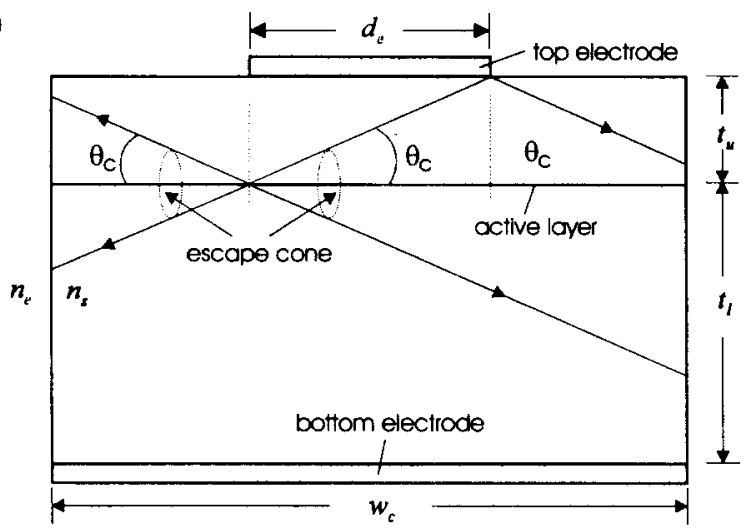

FIG. 6. Shielding of lateral cones by electrodes dependent on the confining layer thickness.

off the center of the top electrode for most photons and therefore the effective diameter of the electrode may be assumed to be smaller than the real diameter $d_{e}$. In this sense, the requirement for $t_{u}$ may be compromised to some degree. A compromised $t_{u}$ may be obtained by considering the case in Fig. 6(b) as

$$
t_{u}=d_{e} \tan \theta_{c}=d_{e} \frac{n_{e}}{\sqrt{n_{s}^{2}-n_{e}^{2}}}
$$

In this regime, only the lateral cone that is directed to the far-side facet of the chip will be partially shielded only in the outer region of the active layer and the other three lateral cones will be completely cleared. The typical $t_{u}$ calculated by Eq.(10) is about $50 \mu m$ and is more easily implemented in practical LEDs. In reality, however, the optimum $t_{u}$ would be between the values by Eqs.(9) and (10). In the case of a bare chip, the $n_{e}$ in Eqs. (9) and (10) should be replaced by the refractive index of the air, $n_{e}=1$, and the optimum $t_{u}$ would be between 30 and $55 \mu \mathrm{m}$. Huang et al. have found that in their InGaAlP LEDs the optimum upper confining layer thicknesses were about $63 \mu \mathrm{m}$ and $45 \mu \mathrm{m}$ for lamp-packaged and bare chip LED, respectively.[6] The comparison of the values indicates that the estimations by Eqs.(9) and (10) are reasonably good.

The lower confining layer thickness $t_{l}$ would be determined similarly by considering the shielding effect by the bottom electrode. Usually the bottom electrode is deposited over the whole bottom surface of the chip. In this case, the diameter of the electrode $d_{e}$ would be the same as the chip size $w_{c}$ and the minimum $t_{l}$ to make all the lateral cones be cleared from the bottom electrode throughout the active layer will be given by

$$
t_{l}=w_{c} \tan \theta_{c}=w_{c} \frac{n_{e}}{\sqrt{n_{s}^{2}-n_{e}^{2}}}
$$

The typical $t_{l}$ evaluated by Eq.(11) is about $120 \mu \mathrm{m}$ and is much larger than the $t_{u}$. One approach to reduce the lower confining layer thickness $t_{l}$ to the level of upper confining layer thickness may be to employ a bottom electrode of island-type as in the top electrode. However, in this case, alignment of both the top and bottom electrodes may be necessary.

If both the upper and lower confining layers are thick enough to satisfy the Eqs.(9) and (11), respectively, the four lateral cones would be completely cleared from the electrodes and thus the space average of the output coupling efficiency of photons, $\eta_{\text {cavg }}$ may be estimated by

$$
\begin{aligned}
\eta_{\text {cavg }} & =\frac{4 \Omega_{c}\left(n_{s}, n_{e}\right)+\eta_{c u} \Omega_{c}\left(n_{s}, n_{e}\right)+\eta_{c d} \Omega_{c}\left(n_{s}, n_{e}\right)}{4 \pi}\left(1-\frac{\left(n_{e}-1\right)^{2}}{\left(n_{e}+1\right)^{2}}\right) \\
& =\frac{1}{2}\left\{4+\left(1+A_{o} / w_{c}^{2}\right)\left(1-\frac{\pi d_{e}^{2}}{4 w_{c}^{2}}\right)\right\}\left(1-\sqrt{1-\left(n_{e} / n_{s}\right)^{2}}\right)\left(1-\frac{\left(n_{e}-1\right)^{2}}{\left(n_{e}+1\right)^{2}}\right)
\end{aligned}
$$

In the above equation, the term $\left(n_{e}-1\right)^{2} /\left(n_{e}+1\right)^{2}$ is due to Fresnel-reflection of escaped photons at the encapsulant-air interface in which almost normal incidence is assumed. In an optically pumped LED, the ohmic electrodes may not be required. In this case, the average output coupling efficiency denoted by $\eta_{\text {copt }}$ would be obtained by substituting the conditions $d_{e}=0, A_{o} / w_{c}^{2}=1$ into Eq.(12) as

$$
\eta_{\text {copt }}=\frac{6 \Omega_{c}\left(n_{s}, n_{e}\right)}{4 \pi}\left(1-\frac{\left(n_{e}-1\right)^{2}}{\left(n_{e}+1\right)^{2}}\right)=3\left(1-\sqrt{1-\left(n_{e} / n_{s}\right)^{2}}\right)\left(1-\frac{\left(n_{e}-1\right)^{2}}{\left(n_{e}+1\right)^{2}}\right)
$$


The average output coupling efficiency $\eta_{\text {cavg }}$ estimated by Eq.(12) is about $24 \%$, with the following device parameters: $w_{c}=216 \mu \mathrm{m}, d_{e}=100 \mu \mathrm{m}$, $A_{o}=w_{c}^{2} / 2, n_{s}=3.50, n_{e}=1.50$. External quantum efficiencies Kish et. al have achieved in their InGaAlP LEDs that are based on both WL and TS schemes are $17.6 \%$ and $23.7 \%$ at wavelengths $629 \mathrm{~nm}$ and $636 \mathrm{~nm}$, respectively. And the corresponding internal quantum efficiencies obtained using the relationship in Eq.(3) are about $72 \%$ and $98 \%$ at wavelengths $629 \mathrm{~nm}$ and $636 \mathrm{~nm}$, respectively and may be estimated a bit high.

The average output coupling efficiency $24 \%$ estimated by Eq.(12) is relatively small compared to $30 \%$ presumed by Kish et al. Even though the Eqs.(12) and (13) have been derived with photon absorption inside the chip ignored, they may still be underestimated. The assumption that all the photons emitted outside escape photons are trapped and absorbed inside the chip may be too stringent. In practical LEDs, some degree of photon trajectory randomization would always occur due to somewhat diffused reflection off the chip-wall which may be better modeled as a kind of a textured surface as a result of either the sawing for chip dicing or possibly some special surfaces treatments. $[7,8]$ These effects would cause some of the photons emitted outside escape cones to couple out of the chip and lead to improved photon output coupling efficiency. Furthermore, in estimating the upward and downward output coupling efficiency in Eqs.(7) and (8), respectively, we assumed all the photons incident on the electrode are absorbed in the ohmic region even though some fraction of photons, in reality, may reflect back into the chip without being absorbed. If we assume that all the photons incident on the electrodes are completely reflected, the average photon output coupling efficiency $\eta_{\text {cavg }}$ would converge to $\eta_{\text {copt }}$ in Eq.(13) which is evaluated to about $28 \%$ in AlGaAs or InGaAlP systems. Thus, in this sense, the average output coupling efficiency expected in the structure based on both WL and TS schemes may be assumed to be in the range between about $24 \%$ and $28 \%$.

The structure described in Fig. 2(b), originally developed by Sugawara et al. for InGaAlP LEDs, utilizes neither WL nor TS and may deserve some discussion. Despite the novelty of the structure, however, the performance may not be very impressive. For instance, as can be seen in Fig. 3, the external quantum efficiency achieved in the structure with both the current blocking layer (CBL) and Braag reflector (BR) is smaller compared to those achieved in InGaAlP LEDs based on either the WL scheme only by Huang et al.[6] or both the WL and TS schemes by Kishi et al.[9,10] The external quantum efficiency achieved, in the other structure by Sugawara et al. based on a $7 \mu m$ thick AlGaAs current spreading layer (CSL) instead of the CBL, is also very poor.[12] It is noted that the comparison would be fair only when the other device parameters includ- ing the internal quantum efficiency are approximately the same in the two devices. The chip dimension $w_{c}$ in the structures by Sugawara is about $400 \mu m$ and is considerably larger than the corresponding values of about 220 and $250 \mu \mathrm{m}$ chosen by Kish et. al. and Huang et al., respectively. In general the effect of internal photon absorption would increase with the chip dimension, because the average pass length traversed by escaped photons would increase with the chip dimension as well. In this sense, the LEDs by Sugawara et al. will have some disadvantage in terms of external quantum efficiency.

The above comparison may indicate that the current spreading by CBL to the outer region of the active layer may not bring a significant benefit compared to the well established WL scheme. Obviously upward cones would be shielded less in the outer region. However, in the case of lateral cones, considerable shielding occurs in the outer region. In reality, the lateral cones are shielded less in the central region when the upper confining layer is relatively thick.[14] In addition, the Bragg reflector (BR) in the structure of Fig. 2(b) may not perform as expected either. The BR that has been proved very useful for coherent waves as in LD may not work so well for incoherent photons with random phases and directions as in LEDs. It is also noted that the BR may possibly be designed such that the high reflectivity is achieved for the photons traveling in the lateral cones as well. A reason for that may be the number of photons traveling in all of the four lateral cones is significantly larger than those traveling in a single downward cone.

\section{INGAN HIGH-BRIGHTNESS LEDS}

In this section InGaN LEDs schematically shown in Fig. 2(c) will be analyzed. One important observation in practical InGaN LEDs is that the overall upper confining layer is less than $1 \mu m$ thick and is far thinner than about at least $130 \mu m$ estimated by, for instance, Eq.(9). Furthermore, a thin ohmic metal is deposited over the whole top surface of the chip. Judging from the conventional design considerations, significant numbers of photons directed either upward or laterally would be shielded by the top electrode and lead to a very poor output coupling efficiency. Considering the presumably high density of defects in InGaN crystals, the internal quantum efficiency would be also very low. The probably poor external quantum efficiency combined with a poor internal quantum efficiency would give rise to an extremely poor external quantum efficiency. In reality, however, the external quantum efficiency achieved in InGaN LEDs is as high as about $9 \%$ and comparable to those achieved in conventional high-brightness LEDs based on both WL and TS schemes. The discrepancy obviously indi- 


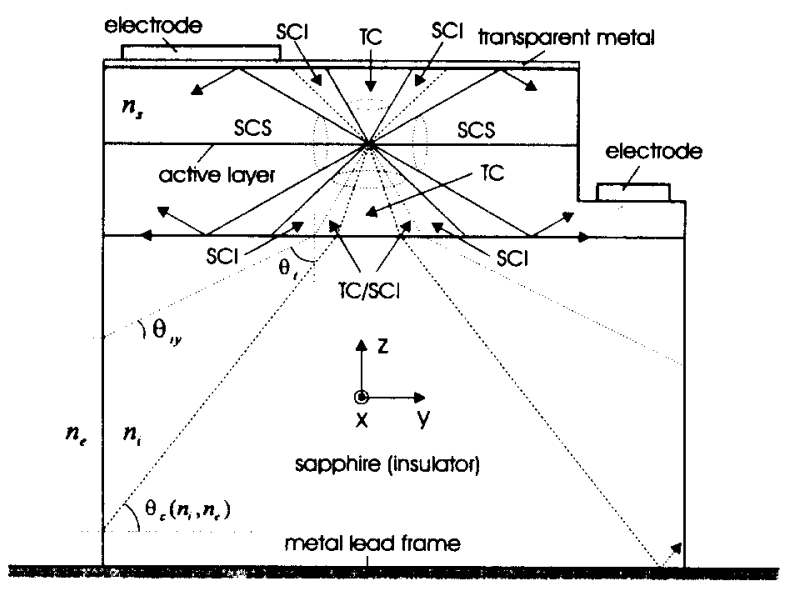

FIG. 7. InGaN LED chip model used for analysis showing various escape cones.

cates that the design rules developed for conventional high-brightness LEDs may not be applicable to InGaN LEDs.

A plausible explanation may start from a basic assumption that the ohmic contact to a wide-bandgap semiconductor[19] such as GaN may be very different from those in other material systems such as GaAlAs, InGaAlP, and $\mathrm{Ga}(\mathrm{As}) \mathrm{P}$. For instance, the GaN crystal near the ohmic contact may remain a regular (not amorphous) crystal leading to negligible photon absorption. This view may be supported by: it is easily observed in InGaN LED lamps that significant amounts of light are being transmitted through the very thin $\mathrm{Ni} / \mathrm{Au}$ ohmic metal deposited over the whole top surface of the chip; 4 ] the thin ohmic metal shows a very fine morphology without any distinctive splotchy pattern often observed in a regular alloyed ohmic contact.[18]

The detailed analysis was described elsewhere.[15] Fig. 7 shows the model we used to estimate the photon output coupling efficiency in InGaN LEDs. In this model, the very thin $\mathrm{Au} / \mathrm{Ni}$ ohmic metal layer deposited over the whole top surface is assumed to be transparent to photons. [4] In addition, the thick ohmic metal pad for bonding wire is assumed to be reflecting all the incident photons regardless of their incidence angles. Based on the model, the behavior of photons emitted into each escape cone is described as follows. First, all the photons emitted inside the lateral cones are assumed to be guided along the slab waveguide which consists of the encapsulant epoxy, semiconductor layer, and sapphire substrate of refractive indices $n_{e}=1.50, n_{s}=2.48, n_{i}=1.78$, respectively, and they would finally couple out of the edge of the chip.

For the case of both the upward and downward cones, however, the situation may be rather complicated as described in Fig. 7. The photons emitted into the region labeled TC would be upward coupled through the very thin transparent ohmic metal either directly or indirectly off the metal lead frame under the chip. Even though some of the photons which are incident perpendicularly on the bonding pad metal may be trapped inside the chip, their contribution might be negligible. In the regime labeled SCI with the incidence angle $\theta_{i}$ in the range

$$
\theta_{c}\left(n_{s}, n_{e}\right)<\theta_{i}<\theta_{c}\left(n_{s}, n_{i}\right)
$$

the photons would be transmitted into the sapphire substrate. In this case, it may be necessary to compare the photon incidence angle to facets of the sapphire insulator with the corresponding critical angle $\theta_{c}\left(n_{i}, n_{e}\right)$ . In reality, the incidence angle has three possibilities, $\theta_{i x}, \theta_{i y}$, and $\theta_{i z}$, i.e. the incidence angle with respect to the plane perpendicular to the $\mathrm{x}$-axis, $\mathrm{y}$-axis, and $\mathrm{z}$-axis respectively. If any of the two incidence angles, $\theta_{i x}$ and $\theta_{i y}$ is smaller than the critical angle $\theta_{c}\left(n_{i}, n_{e}\right)$ , the photon would have a chance to couple out of the chip. The angle $\theta_{i z}$ need not be considered because all the photons incident on the bottom of the chip would be reflected back into the chip off the metal lead frame. In order to find the maximum value of the smaller of two incidence angles, $\theta_{i x}$ and $\theta_{i y}$, we need to consider only the photons with the minimum incidence angle $\theta_{i}=\theta_{c}\left(n_{s}, n_{e}\right)$ in Eq.(14). The photons will be transmitted into the sapphire substrate with the transmitted angle $\theta_{t}$ which is to be evaluated by the Snell's law $[17]$

$$
n_{i} \sin \theta_{t}=n_{s} \sin \left(\theta_{c}\left(n_{s}, n_{e}\right)\right)=n_{e}
$$

The direction cosines of the transmitted photon, $m_{x}, m_{y}$, and $m_{z}$ would be given by

$$
\begin{array}{r}
m_{x}=\cos \theta_{i x}, \quad m_{y}=\cos \theta_{i y}, \quad m_{z}=-\cos \theta_{t} \\
m_{x}^{2}+m_{y}^{2}+m_{z}^{2}=1
\end{array}
$$

Both $m_{x}$ and $m_{y}$ in Eqs.(16) and (17) would not be determined until the azimuth direction of the photons is specified. The maximum value of the smaller of two incidence angles, $\theta_{i x}$ and $\theta_{i y}$ would result when the photon travels along the azimuth direction which is $45^{\circ}$ off from the $\mathrm{x}$-axis with the condition

$$
m_{x}^{2}=m_{y}^{2}
$$

satisfied. Using the Eqs.(15) - (18), the maximum value of the smaller of two incidence angles, $\theta_{i x}$ and $\theta_{i y}$ is evaluated to about $53.4^{\circ}$ and is smaller than the critical angle $\theta_{c}\left(n_{i}, n_{e}\right)=57.4^{\circ}$. This implies that all the photons emitted into the regime labeled SCI in Fig. 6 would be coupled out through side facet of the insulator substrate. It is quite obvious that the photons emitted into the region labeled TC/SCI which is inside the region SCI would be coupled out either through side facet of the substrate or through the top surface, depending on the azimuthal photon direction in the 
$x-y$ plane.

Based on the above reasoning, the average photon

output coupling efficiency $\eta_{\text {cavg }}$ would be estimated by

$\eta_{\text {cavg }}=\frac{4 \Omega_{c}\left(n_{s}, n_{e}\right)+2 \Omega_{c}\left(n_{s}, n_{i}\right)}{4 \pi}\left(1-\frac{\left(n_{e}-1\right)^{2}}{\left(n_{e}+1\right)^{2}}\right)=\left(3-2 \sqrt{1-\left(n_{e} / n_{s}\right)^{2}}-\sqrt{1-\left(n_{i} / n_{s}\right)^{2}}\right)\left(1-\frac{\left(n_{e}-1\right)^{2}}{\left(n_{e}+1\right)^{2}}\right)$

where we assume that all the photons inside the four lateral cones as well as both the upward and downward cones with the same solid angle $\Omega_{c}\left(n_{s}, n_{i}\right)$ couple out of the chip. The $\eta_{\text {cavg }}$ is evaluated to about $68 \%$ and is about $2.5-2.8$ times that of $24-28 \%$ in $\mathrm{AlGaAs}$ or InGaAlP high-brightness LEDs estimated by Eq.(12) or (13). Note that the significant improvement of the output coupling efficiency in InGaN LEDs is largely due to the enlarged escape cone solid angle $\Omega_{c}\left(n_{s}, n_{e}\right)$ as a result of better index-matching to the encapsulant epoxy. For instance, $\Omega_{c}\left(n_{s}, n_{e}\right)$ in InGaN LEDs is about 1.28 steradians and is about 2.1 times that in AlGaAs or InGaAlP LEDs. The absolute values of output coupling efficiency estimated by either Eq.(12) or (19) may involve a significant error due to the incomplete assumptions regarding the internal photon absorption and photon trajectory randomization. However, considering that the same assumptions are made in estimating the output coupling efficiencies, the error involved in the ratio of the output coupling efficiencies may be much smaller.

Once the average photon output coupling efficiency $\eta_{\text {cavg }}$ is obtained, the internal quantum efficiency $\eta_{i}$ which is not easily measured in LEDs would be estimated by using the relationship in Eq.(2). Some of the best internal quantum efficiencies in InGaN LEDs are $9.2 \%, 9.1 \%, 6,3 \%$, and $1.2 \%$ at wave lengths $405 \mathrm{~nm}, 450 \mathrm{~nm}, 520 \mathrm{~nm}$, and $590 \mathrm{~nm}$, respectively.[25] And the corresponding internal quantum efficiencies are $13.5 \%, 13.3 \%, 9.2 \%$, and $1.8 \%$, respectively. It is noted that in the calculation we have used the same photon output coupling efficiency $\eta_{\text {cavg }}$.

\section{CONCLUSION}

In conclusion, based on the escape cone concepts, we have analyzed high-brightness LEDs. In AlGaAs or InGaAlP LEDs, photon absorption in the ohmic region under the electrode is known to be significant and therefore photon shielding by the electrodes should be minimized. The shielding effect of the upward cone is rather insensitive to the upper confining layer thickness while that of the lateral cones decreases as the upper confining layer thickness. The optimum WL thickness, which decreases in general as the chip size $w_{c}$ or the top electrode diameter $d_{e}$ decreases, is typically as thick as $50 \mu \mathrm{m}$. Reduced chip dimension will decrease the total pass length traversed by the escaped photons in the output coupling process. This effect will minimize the internal photon absorption and leads to improved external quantum efficiency. In these senses, the approach to increase the brightness or output power by increasing chip size will not be very effective.

The design rules for the upper or lower confining layer thickness that has been found to work reasonably well for either AlGaAs or InGaAlP LEDs is not applicable to InGaN LEDs. Photon absorption in the region near the ohmic contact to a very wide bandgap semiconductor such as $\mathrm{GaN}$ may be negligible. In this case, the ohmic contact may act as a reflector for incoming photons. Thus, the significant photon shielding as a result of a thin upper confining layer in practical InGaN LEDs may not considerably degrade the photon output coupling efficiency. The photon output coupling efficiency estimated in InGaN LEDs is about $68 \%$ and is about $2.5-2.8$ times that of the conventional high-brightness LED structures based on both WL and TS schemes. Furthermore, the various semiconductor layers in InGaN LEDs are less than 5 $\mu m$ and are extremely thin compared to about $150 \mu m$ typical in conventional high-brightness LEDs. The tiny semiconductor volume, as a result of thin layers, may be another important factor to minimize the internal photon absorption. The impressive output coupling efficiency as well as the minimized internal photon absorption should lead to the remarkably high external quantum efficiency, despite the presumably very low internal quantum efficiency.

\section{ACKNOWLEDGMENTS}

The author would like to thank Director Y. S. Jeon of AP Electronics for providing various high-brightness LED lamps and helpful discussions. The author also acknowledges the support of Korea Ministry of Education through Inter-university of Semiconductor Research Center (ISRC-96-E-3018) in Seoul National University and the partial support of the University Basic Research Program sponsored by Korea Ministry of Information and Communication.

\section{REFERENCES}

[1] S. Nakamura, T. Mukai, and M. Senoh, Appl. Phys. Lett. 64, 1687 (1994). S. Nakamura, M. Senoh, N. Iwasa, and S. Nagahama, Appl. Phys. Lett. 67, 1868 (1995). 
[2] S. Nakamura, M. Senoh, N. Iwasa, and S. Nagahama, T. Yamada, and T. Mukai, Japan. J. Appl. Pyhs. Lett. 34, L1332 (1995).

[3] S. Nakamura, M. Senoh, N. Iwasa, and S. Nagahama, Japan. J. Appl. Phys. Lett. 34, L797 (1995).

[4] S. Nakmura and G. Fasol, "The blue laser diode GaN based light emitters and lasers", (Springer, Berlin, 1997).

[5] R. Fletcher, C. Kuo, T. Osentowski, J. Yu, and V. Robbins, Hewlett-Packard J. 6 (1993).

[6] K. Huang, J. Yu, C. Kuo, R. Fletcher, T. Osentowski, L. Stinson, M. Craford, Appl. Phys. Lett. 61, 1045 (1992).

[7] I. Schnitzer, E. Yablonovitch, C. Caneau, T. Gmitter, and A. Scherer, Appl. Phys. Lett. 62, 131 (1993).

[8] I. Schnitzer, E. Yablonovitch, C. Caneau, T. Gmitter, and A. Scherer, Appl. Phys. Lett. 63, 2174 (1993).

[9] F. Kishi, F. Steranka, D. DeFevere, D. Vanderwater, K. Park, C. Kuo, T. Osentowski, M. Peanasky, J. Fletcher, D. Steigerwald, and M. Craford, Appl. Phys. Lett. 64, 2839 (1994).
[10] F. Kishi, D. Vanderwater, D. DeFevere, D. Steigerwald, G. Hofler, K. Park, and F. Steranka, Electron. Lett. 32, 132 (1996).

[11] H. Sugawara, K. Itaya, H. Nozaki, and G. Hatakoshi, Appl. Phys. Lett. 61, 1775 (1992).

[12] H. Sugawara, M. Ishikawa, and G. Hatakoshi, Appl. Phys. Lett. 58, 1010 (1991).

[13] D. Eason, Z. Yu, W. Hughes, W. Roland, C. Boney, J. Cook, J. Schetzina, G. Cantwell, and W. Harsch, Appl. Phys. Lett. 66, 115 (1995).

[14] S. Lee, Japan. J. Appl. Phys., 37, in printing (1997).

[15] S. Lee, submitted to Electron. Lett. (1997).

[16] C. Jacoboni and P. Lugli, "The Monte Carlo method for semiconductor device simulation," (Springer, New York, 1989).

[17] M. Born and E. Wolf, "Principles of optics," (Pergamon, Oxford, 1975).

[18] R. Williams, "Gallium arsenide processing techniques," (Artech Houuse, Dedham, 1984). H. Morkoc, S. Strite, G. Gao, M. Lin, B. Sverdlov, and M. Burns, J. Appl. Phys. 76, 1363 (1994). 